\title{
Conditioning of digitally modulated signals backscattered from a tag to a reader
}

\author{
Oleg Smirnov*, Evgeniy Bogatyrev, and Sergey Smolskiy \\ National Research University "Moscow Power Engineering Institute", \\ 111250 Krasnokazarmennaya, 14, Moscow, Russia
}

\begin{abstract}
Results of modeling of the signal passing through equivalent circuits with the time-varying parameter are given in this paper. Parameters of such circuits correspond to the reflection coefficient, which is necessary for modulation of the backscattered signal in the tag-to-reader line. The main idea of this research is developing a general description of conditioning of digitally modulated signals backscattered from a tag to a reader.
\end{abstract}

\section{Introduction}

For a long time, RFID was the only technology where backscattering modulation (BM) used for data transmission. However, last several years one can observe an increase of interest to such kind of signal conditioning. Backscattering modulation finds its implementation in such data transmission systems (DTS) as BLE [1], Wi-Fi [2], LoRA [3, 4], WLAN [5] and GSM. It also appears in IoT [6] and WSN [7]. The usage of BM out of RFID requires the implementation of more sophisticated signals than ASK-2 and PSK-2 which are typical for this technology. This reason determines the necessity of the development of the general theory for generation of signals backscattered from a tag to a reader.

Nevertheless, there are a lot of publications dedicated to the usage of the backscattering modulation in different data transmission system but the signal generation process was viewed partially. They considered the modulation type and modulator design escaping the connection of backscattered signal and device parameters. Physical limitations and physical feasibility conditions are also missing.

To sum up, one can say there is no universal way to describe signal conditioning of the modulated backscattered signal that could connect such signal parameters as a phase and an amplitude with tag's modulator parameters.

\section{Background}

There are different modulation types that were realized in related works [8]. The authors found examples of modulators of backscattering signal for PSK, ASK, FSK-OOK, FSK-

*Corresponding author: oleg.vladimirovich.smirnov@gmail.com 
ASK, and QAM-4, QAM-8, QAM-16. Despite of the fact that lots variants of modulators were realized, just a few publications explain the connection of signal parameters with a modulator.

The power of the backscattered signal received by the reader describes as:

$$
P_{B S N}=\frac{P_{T} G_{R, T} G_{R, R}}{(4 \pi)^{3} r^{4}} \sigma_{N},
$$

where $P_{T}$ is a power transmitted by the reader, $G_{R, T}$ is a gain of transmitting antenna and $G_{R, R}$ is a gain of receiving antenna, $r$ is a distance between a tag and a reader, $\sigma_{N}$ is radar cross-section (RCS) of the tag's antenna that corresponds to the following equation

$$
\sigma_{N}=\frac{\lambda^{2} G_{t}^{2}}{4 \pi}\left|A_{S}+\Gamma_{N}^{*}\right|^{2}
$$

Here $G_{t}$ is the tag's antenna gain, $\lambda$ is a wavelength, $A_{S}$ is the structural component determined by the structure of the tag antenna and $\Gamma_{\mathrm{N}}{ }^{*}$ is a conjugated reflection coefficient that could be calculated as

$$
\Gamma_{N}^{*}=\frac{Z_{A}^{*}-Z_{L, N}}{Z_{A}+Z_{L, N}},
$$

where $Z_{A}$ is the impedance of the tag's antenna, $Z_{L, N}$ is the load impedance that determines transmitting of the $N$ symbol. The load impedance is the main component affecting the backscattered signal.

The main idea of the backscattered signal modulation is the changing of the load impedance for reaching the necessary modulation state. Despite the fact that strict connection between the backscattered signal and the load impedance exists, there are only two publications $[9,10]$, as we know, where impedance values were determined for the transmitting symbol.

In addition, there is no model what connects the time-domain representation of a signal transmitting from a tag to a reader and the load impedance. Existing models allows only determine the signal convolution with the impulse response function of a channel missing the dynamics of the conjugate reflection coefficient. This work presents the model that establishes the connection of the time-domain representation of the backscattered signal and the load impedance.

\section{Signal model}

\subsection{Signal received by a reader}

In previous work [11], authors have expressed an equation for the open-circuit voltage induced at the terminals of the reader that received after interaction of the unmodulated signal traveling from a reader with a tag in the backscatter system shown at Fig. 1:

$$
\begin{aligned}
& V_{r e c}\left(\boldsymbol{r}_{T R}, t\right)=-\left(\frac{\mu}{4 \pi}\right)^{2} \frac{1}{4 R_{A}^{R} r_{R T} r_{T R} Z_{0}^{R}}\left[\boldsymbol{h}_{R}^{t}\left(\boldsymbol{e}_{r_{R T}}, \cdot\right) \otimes \boldsymbol{h}_{R}^{r}\left(\boldsymbol{e}_{r_{R T}}, \cdot\right) \otimes \boldsymbol{h}_{T}^{r}\left(\boldsymbol{e}_{r_{T R}}, \cdot\right) \otimes\right. \\
& \left.\otimes \boldsymbol{h}_{T}^{t}\left(\boldsymbol{e}_{r_{T R}}, \cdot\right) \otimes\left\{\left[A_{S}(\cdot)+\Gamma^{*}(\cdot)\right] \otimes V_{g}(\cdot) \otimes\left[1-\Gamma^{t}(\cdot)\right]\right\}\right]\left(t-t_{g}-\frac{r_{R T}}{c}-\frac{r_{T R}}{c}\right),
\end{aligned}
$$

where $\boldsymbol{r}_{\boldsymbol{T}}, \boldsymbol{r}_{\boldsymbol{R} T}$ are radius-vectors of distance from a tag to a reader and from the reader to the tag, respectively, $\boldsymbol{e}_{r_{T R}}$ and $\boldsymbol{e}_{\boldsymbol{r}_{R T}}$ are their eigenvectors, $r_{T R}, r_{R T}$ are modules of radiusvectors, $\boldsymbol{h}_{R}^{t}, \boldsymbol{h}_{R}^{r}$ are effective heights of the reader in radiation and transmission mode 
respectively, $\boldsymbol{h}_{T}^{r}, \boldsymbol{h}_{T}^{t}$ are similar effective heights for the tag, $V_{g}$ is the voltage induced by a reader's generator with impedance $Z_{0}^{R}, t_{g}$ is the time delay of the signal travelling from the generator to an antenna through the transmission line of the reader, $\Gamma^{t}$ is the reflection coefficient looking into the generator of the radiating antenna circuit (the reader), $c$ is the speed of light. Here we use the symbol notation like $[f(\cdot) \otimes g(\cdot)](\tau)=\int f(t) g(t-\tau) d \tau$ for determining of the convolution operation.
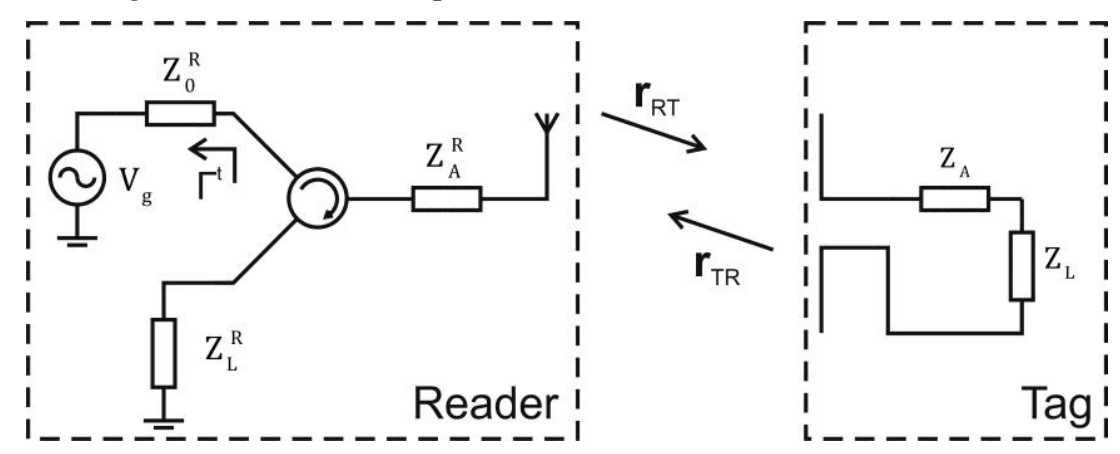

Fig. 1. The backscatter system.

\subsection{Law of reflection coefficient}

\subsubsection{Data component}

From (4) follows that the main influence on a signal scattering from a tag to a reader is determined by the normalized function:

$$
v_{\text {mod }}(t)=\int_{0}^{t}\left[1+\Gamma^{*}(\tau)\right] V_{g}(t-\tau) d \tau
$$

Here, we suppose that the transmission line of the reader is matched, $\tau=t-t_{g}-r_{R T} / c-$ $r_{T R} / c, A_{S}=1$, antennas of a reader and a tag are isotropic, effective heights are constant and it doesn't affect signal propagation. This equation is the convolution of two functions. Due to the linear property of the convolution operator, we can rewrite (5) as

$$
v_{\text {mod }}(t)=\int_{0}^{t} V_{g}(t-\tau) d \tau+\int_{0}^{t} \Gamma^{*}(\tau) V_{g}(t-\tau) d \tau=v_{\text {struct }}(t)+v_{\text {data }}(t) .
$$

The left side of (6) is the structural component which could include different additional reflections what we will not consider in the following analysis. Thus, data $v_{\text {mod }}$ defines only by the data component:

$$
v_{\text {data }}(t)=\int_{0}^{t} \Gamma^{*}(\tau) V_{g}(t-\tau) d \tau
$$

Owing to the convolution theorem, the image of (7) corresponds to:

$$
v_{\text {data }}(s)=\Gamma^{*}(s) V_{g}(s) \text {. }
$$

So if we know the law of the image which determines the data component, we can define the law of reflection writing the inverse Laplace transform of (8):

$$
\Gamma^{*}(t)=L^{-1}\left\{\frac{V_{\text {data }}(s)}{V_{g}(s)}\right\} .
$$




\subsubsection{Pulse conditioning}

As an example, let us consider radio impulse conditioning. Data component should be like:

$$
A(t)=[\sigma(t)-\sigma(t-T)] e^{j \omega_{0} t},
$$

where $T$ is the time length of the pulse with the carrier frequency $\omega_{0}$. The image of this equation corresponds to:

$$
A(s)=\left(1-e^{-s T} e^{j \omega_{0} T}\right) \frac{s+j \omega_{0}}{s^{2}+j \omega_{0}^{2}} .
$$

According to (9), the reflection coefficient should be determined as:

$$
\Gamma^{*}(s)=1-e^{-s T} e^{j \omega_{0} T} .
$$

It could be rewritten as:

$$
\Gamma^{*}(s)=e^{-s 0 T} e^{j \omega_{0} 0 T}-e^{-s T} e^{j \omega_{0} T} .
$$

This equation corresponds to the inverse Laplace transform of:

$$
\Gamma^{*}(t)=\delta(t-0 T) e^{j \omega_{0} 0 T}-\delta(t-T) e^{j \omega_{0} T} .
$$

In this way, the impulse sequence that could be described as

$$
A(t)=\sum_{N=0}^{M} A_{N}\{\sigma(t-N T)-\sigma[t-(N+1) T]\} e^{j \omega_{0} t},
$$

where $A_{N}$ is the amplitude of the transmitting symbol, $T$ is the period of the pulse sequence, $N$ is a number of transmitting symbol. The law of the reflection coefficient for (14) determined as

$$
\Gamma^{*}(t)=\sum_{N=0}^{M} A_{N}\left\{\delta(t-N T) e^{j \omega_{0} N T}-\delta[t-(N+1) T] e^{j \omega_{0}(N+1) T}\right\},
$$

The Laplace transform of (16) can be described by

$$
\Gamma^{*}(s)=\sum_{N=0}^{M}\left(A_{N} e^{-s N T} e^{j \omega_{0} N T}-A_{N} e^{-s(N+1) T} e^{j \omega_{0}(N+1) T}\right) .
$$

The resulting expression may be used for the description of the law reflection coefficient which is necessary for the conditioning of a signal with ASK modulation which could be recorded as:

$$
m_{A S K}(t)=\operatorname{Re}\left[\sum_{N=0}^{M} A_{N}\{\sigma(t-N T)-\sigma[t-(N+1) T]\} e^{j \omega_{0} t}\right],
$$

This signal is corresponding to the data component that can be expressed as

$$
v_{\text {data }}(t)=\int_{0}^{t}\left(\sum_{N=0}^{M} A_{N}\left\{\delta(t-N T) e^{j \omega_{0} N T}-\delta[t-(N+1) T] e^{j \omega_{0}(N+1) T}\right\}\right) e^{j \omega_{0}(t-\tau)} d \tau .
$$

According to (17), the image of (19) may be represented as

$$
v_{\text {data }}(s)=\sum_{N=0}^{M}\left(A_{N} e^{-s N T} e^{j \omega_{0} N T}-A_{N} e^{-s(N+1) T} e^{j \omega_{0}(N+1) T}\right) \frac{s+j \omega_{0}}{s^{2}+j \omega_{0}^{2}} .
$$

That could be rewritten as a difference

$$
v_{\text {data }}(s)=\sum_{N=0}^{M} A_{N} e^{j \omega_{0} N T} e^{-s N T} \frac{s+j \omega_{0}}{s^{2}+j \omega_{0}^{2}}-\sum_{N=0}^{M} A_{N} e^{j \omega_{0}(N+1) T} e^{-s(N+1) T} \frac{s+j \omega_{0}}{s^{2}+j \omega_{0}^{2}},
$$

We make the following change of variables:

$$
\begin{gathered}
F_{N}(s)=e^{j \omega_{0} N T} e^{-s N T} \frac{s+j \omega_{0}}{s^{2}+j \omega_{0}^{2}} \mapsto F_{N}(t)=e^{j \omega_{0}(t-N T)} e^{j \omega_{0} N T} \sigma(t-N T)=e^{j \omega_{0} t} \sigma(t-N T) \\
F_{N+1}(s)=e^{j \omega_{0}(N+1) T} e^{-s(N+1) T} \frac{s+j \omega_{0}}{s^{2}+j \omega_{0}{ }^{2}} \mapsto F_{N+1}(t)=e^{j \omega_{0} t} \sigma[t-(N+1) T]
\end{gathered}
$$


Then we have

$$
v_{\text {data }}(t)=\sum_{N=0}^{M} L^{-1}\left(A_{N} F_{N}(s)\right)-\sum_{N=0}^{M} L^{-1}\left(A_{N} F_{N+1}(s)\right) .
$$

Similar to (15)-(23), we can reach equations for PSK and QAM-M. In this case, the expression determining the signal with PSK modulation

$$
s_{P S K}(t)=\operatorname{Re}\left[\sum_{N=0}^{M} e^{j \varphi_{N}}\{\sigma(t-N T)-\sigma[t-(N+1) T]\} e^{j \omega_{0} t}\right]
$$

will be corresponded to the data component

$$
v_{\text {data }}(t)=\sum_{N=0}^{M} L^{-1}\left(e^{j \varphi_{N}} F_{N}(s)\right)-\sum_{N=0}^{M} L^{-1}\left(e^{j \varphi_{N}} F_{N+1}(s)\right) .
$$

For signal with QAM-M

$$
s_{Q A M}(t)=\operatorname{Re}\left[\sum_{N=0}^{M} A_{N} e^{j \varphi_{N}}\{\sigma(t-N T)-\sigma[t-(N+1) T]\} e^{j \omega_{0} t}\right]
$$

we can match the expression

$$
v_{\text {data }}(t)=\sum_{N=0}^{M} L^{-1}\left(A_{N} e^{j \varphi_{N}} F_{N}(s)\right)-\sum_{N=0}^{M} L^{-1}\left(A_{N} e^{j \varphi_{N}} F_{N+1}(s)\right) .
$$

We will replace $A_{N} e^{j \varphi_{N}}$ by the complex-conjugate reflection coefficient $\Gamma^{*}{ }_{N}$ :

$$
v_{\text {data }}(t)=\sum_{N=0}^{M} L^{-1}\left(\Gamma_{N}^{*} F_{N}(s)\right)-\sum_{N=0}^{M} L^{-1}\left(\Gamma_{N}^{*} F_{N+1}(s)\right) .
$$

This expression is valid only when the reflection coefficient is not a frequency-dependent function. For the real case, we should consider $\Gamma^{*}{ }_{N}$ as

$$
\Gamma_{N}^{*}(\omega)=\frac{Z_{A}^{*}(\omega)-Z_{L, N}(\omega)}{Z_{A}(\omega)+Z_{L, N}(\omega)} .
$$

This equation corresponds to the Laplace form

$$
\Gamma_{N}^{*}(s)=\frac{Z_{A}^{*}(s)-Z_{L, N}(s)}{Z_{A}(s)+Z_{L, N}(s)} .
$$

Then, the time-domain representation of the data component could be written as

$$
v_{\text {data }}(t)=\sum_{N=0}^{M} L^{-1}\left(\Gamma_{N}^{*}(s) F_{N}(s)\right)-\sum_{N=0}^{M} L^{-1}\left(\Gamma_{N}^{*}(s) F_{N+1}(s)\right) .
$$

Therefore the data component determines as a sum of reactions of an equivalent circuit with the transmission coefficient $\Gamma_{N}^{*}(\omega)$ which corresponds to the transmitting symbol on the harmonic signal limited by the step function. The result of the passing of such input impact can be easily calculated by popular math software.

\subsubsection{Load impedance calculation}

One can determine the tag's load impedance setting the required values of $\Gamma_{N}^{*}(\omega)$ :

$$
Z_{L, N}=\frac{Z_{A}^{*}-Z_{A} \Gamma_{N}^{*}}{1+\Gamma_{N}^{*}} .
$$

In reality, the load impedance consists of the modulating impedance and the shunting impedance that is represented by different tag's blocks what also connected to the tag's antenna terminals such as a demodulator and energy harvester. In this case, the equivalent circuit of the tag will have the form shown in Fig. 2. The case when shunting blocks don't 
exist or do not affect the reflection coefficient can also occur, but we will not consider this case in this paper.

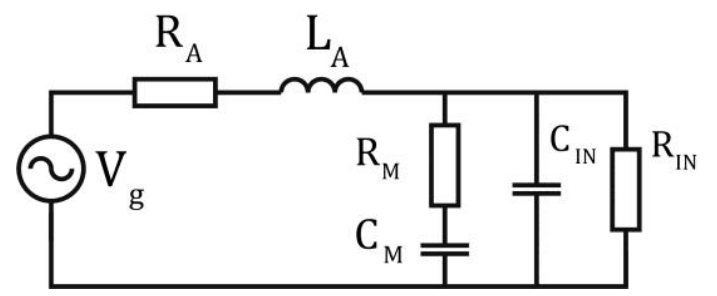

Fig. 2. The equivalent circuit of the tag.

Mutual arrangement (serial or parallel) of pairs of $R_{M}$ and $C_{M}$ or $C_{I N}$ and $R_{I N}$ does not influence further reasoning. The total conductivity of the load will be determined as

$$
G_{L}=G_{M}+g_{I N}+j \omega C_{I N} .
$$

where $g_{I N}=1 / R_{I N}$ and the modulating impedance $G_{M}=\left(Z_{M}\right)^{-1}=\left(R_{M}+1 / j \omega C_{M}\right)^{-1}$. In further analysis, we will assume that $g_{I N}$ and $C_{I N}$ are known in advance and determined by the tag's manufacturer and corresponds to the antenna matching mode. Thus, we obtain the following expressions describing the modulating conductivity and capacitance, respectively:

$$
\begin{gathered}
R_{M}=\operatorname{Re}\left\{\left[G_{L}-\left(g_{I N}+j \omega C_{I N}\right)\right]^{-1}\right\}=\frac{\operatorname{Re}\left(G_{L}\right)-g_{I N}}{\left[\operatorname{Re}\left(G_{L}\right)-g_{I N}\right]^{2}+\left[\operatorname{Im}\left(G_{L}\right)-j \omega C_{I N}\right]^{2}}, \\
C_{M}=\left(\omega_{0} \operatorname{Im}\left\{\left[G_{L}-\left(g_{g x}+j \omega C_{I N}\right)\right]^{-1}\right\}\right)^{-1}=\frac{\left[\operatorname{Re}\left(G_{L}\right)-g_{I N}\right]^{2}+\left[\operatorname{Im}\left(G_{L}\right)-j \omega C_{I N}\right]^{2}}{\omega_{0}\left[\operatorname{Im}\left(G_{L}\right)-j \omega C_{I N}\right]} .
\end{gathered}
$$

The expressions obtained allow us determine the necessary values of the modulating capacitance and modulating resistance.

\subsection{Physical feasibility}

The relations $(33,34)$ impose the following requirement on physical feasibility: modulating resistance and modulating capacitance should only take positive values. As an example, we will use input the impedance of a tag Monza 4QT. The manufacturer gives the following values of the parameters of the RFID tag for the operating frequency $\omega_{0}=866 \mathrm{MHz}: C_{I N}=$ $p F, R_{I N}=1650 \mathrm{Ohm}, R_{A}=13 \mathrm{Ohm}, L_{A}=27.75 \mathrm{nH}$. Let's suppose that the complex conjugate coefficient $\Gamma^{*}$ should equal to $0.5 \mathrm{e}^{\mathrm{j} \pi / 4}$. For the given complex conjugate reflection coefficient, the real and imaginary parts of the load conductivity will be $\operatorname{Re}\left(G_{L}\right)=0.22 \mathrm{mS}$ and $\operatorname{Im}\left(G_{L}\right)=6.42 \mathrm{mS}$, respectively. For these values, we can calculate

$$
\begin{gathered}
R_{M}=-2.19 \mathrm{kOhm} \\
C_{M}=-0.2 \mathrm{pF}
\end{gathered} .
$$

From (36), it is seen that the modulating conductivity and capacitance is a negative number, which is unacceptable under given conditions. Let us determine the permissible values of the reflection coefficient at which the values of the modulating resistance and capacitance satisfy the given requirements. We will plot them depending on the module and the phase of the reflection coefficient (see Fig. 2 and Fig. 3).

From the obtained dependencies (see Fig. 2, 3) it is seen that there is only a certain range of values is feasible for given limitations. For a given conditions one can realize a modulator with the phase shift for 135 degrees and variation of the module of the reflection coefficient from 0 to 0.83 . Let's analyze the results. 


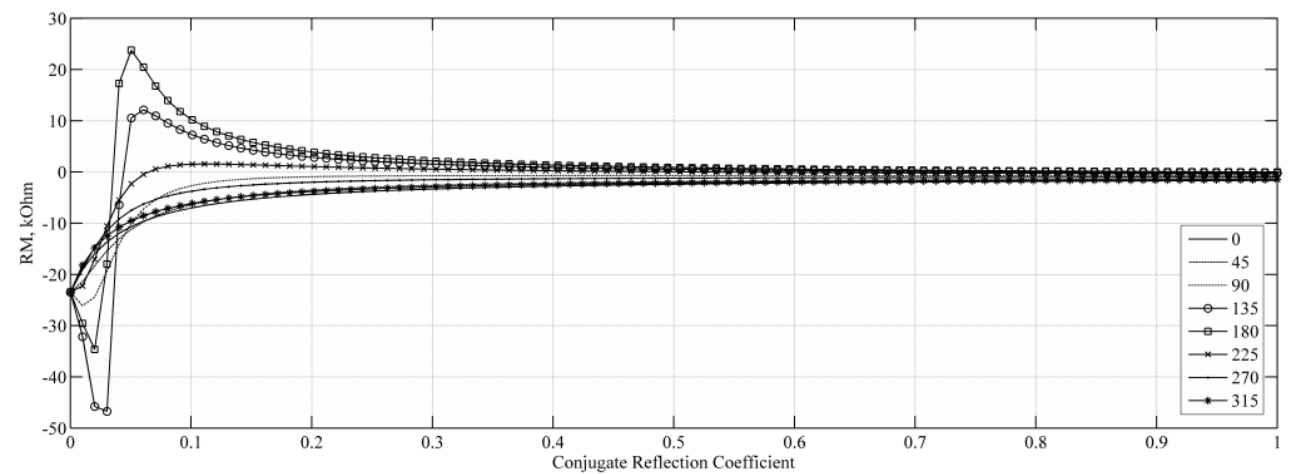

Fig. 2. Modulating resistance against amplitude and phase of the reflection coefficient

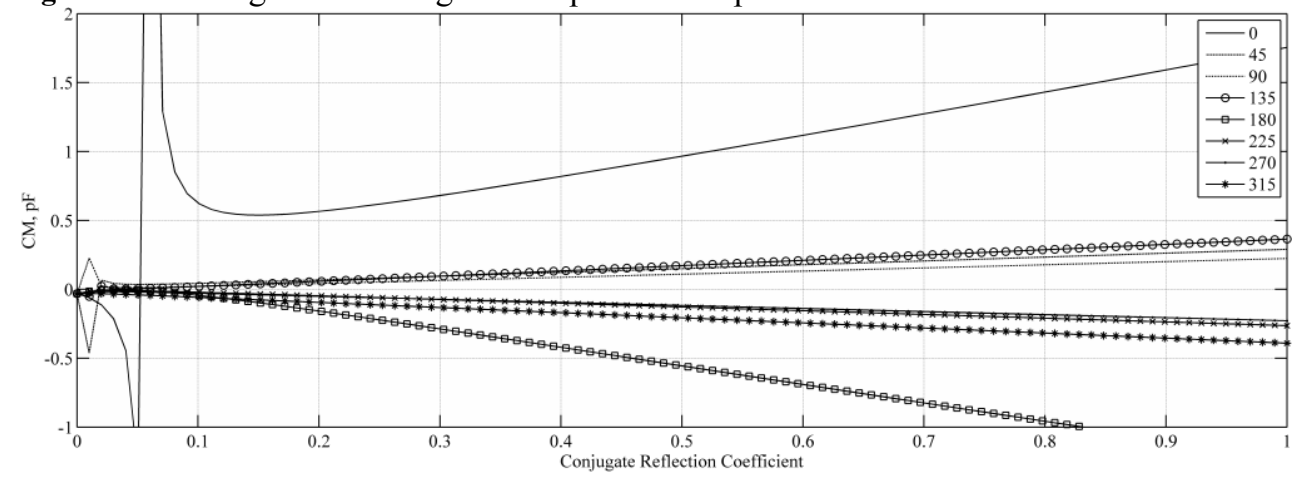

Fig. 3. Modulating capacitance against amplitude and phase of the reflection coefficient

Modulating impedance is defined as:

$$
Z_{M}=R_{M}+\frac{1}{j \omega C_{M}}=\frac{1}{\operatorname{Re}\left(G_{L}\right)+j \operatorname{Im}\left(G_{L}\right)-\left(g_{I N}+j \omega C_{I N}\right)} .
$$

Decomposing (37) for quadrature components we will get that one have to comply with the following conditions to ensure physical feasibility:

$$
R_{I N}>\frac{1}{\operatorname{Re}\left(G_{L}\right)} ; C_{I N}<\frac{\operatorname{Im}\left(G_{L}\right)}{\omega} .
$$

\section{Signal conditioning}

As an example, let us analyze the ASK modulating signal. Symbol "0" corresponds to low level of the backscattered signal and " 1 " to the high level. Bit length will equal to $T=0.1$ $\mu s$, the bit rate is $10 \mathrm{Mbit} / \mathrm{s}$. Such bit length were chosen to make equations faster, typical bit length in UHF RFID system is $25 \mu \mathrm{s}$. Low and high level of the backscattered signal corresponds to the low and high value of the module of the conjugate reflection coefficient respectively. We will use the circuit from Fig. 1 with Monza 4QT impedance at the central frequency at $866 \mathrm{MHz}$.

High level of the conjugate reflection coefficient corresponds to $\Gamma^{*}=0.8 \mathrm{e}^{\mathrm{j} 3 \pi / 4}$. For this value, the modulating resistance and capacitance will equal $R_{M}=3.2 \mathrm{Ohm}$ and $C_{M}=0.287$ $p F$ respectively. The low level of the conjugate reflection coefficient is defined by $\Gamma^{*}=$ $0.1 \mathrm{e}^{\mathrm{j} 3 \pi / 4}$. Modulating resistance and capacitance will equal $R_{M}=7354 \mathrm{Ohm}$ and $C_{M}=20.86$ $f F$ respectively.

The load impedance for the circuit in Fig. 1 can be calculated by this equation. 


$$
Z_{L}(\omega)=\frac{R_{I N}\left(1+j \omega C_{M} R_{M}\right)}{1-C_{I N} R_{I N} C_{M} R_{M} \omega^{2}+j \omega R_{I N} C_{I N}+j \omega C_{M} R_{M}+j \omega C_{M} R_{I N}}
$$

According to (29), an operator form of the (38) is defined as:

$$
Z_{L}(s)=\frac{R_{I N}\left(1+s C_{M} R_{M}\right)}{1+C_{I N} R_{I N} C_{M} R_{M} s^{2}+s R C+s C_{M} R_{M}+s C_{M} R_{I N}} .
$$

Using (40), (31) and (30) one can find the reactions of the equivalent circuit. The timedomain representation for a test sequence is shown in Fig. 3. The spectrum of this signal is shown in Fig. 4.

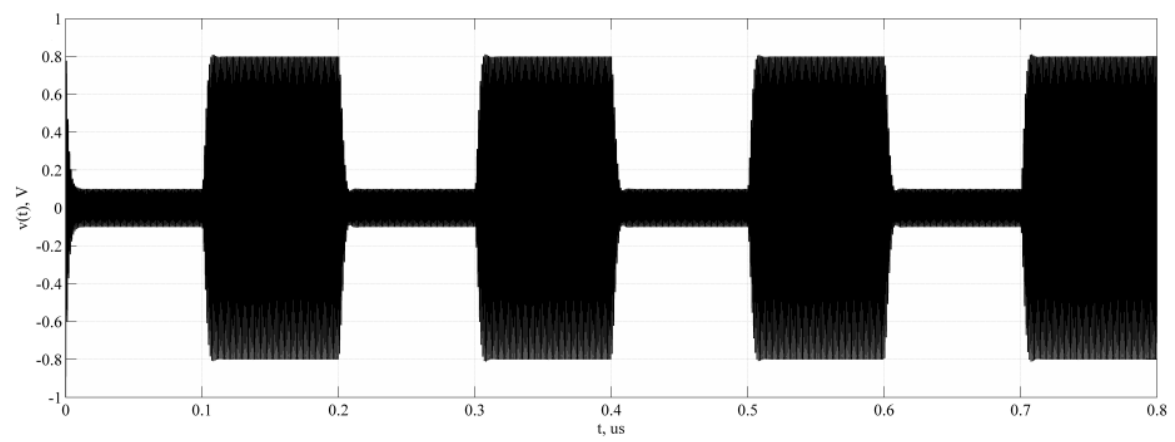

Fig. 3. The time-domain representation for the test sequence with ASK

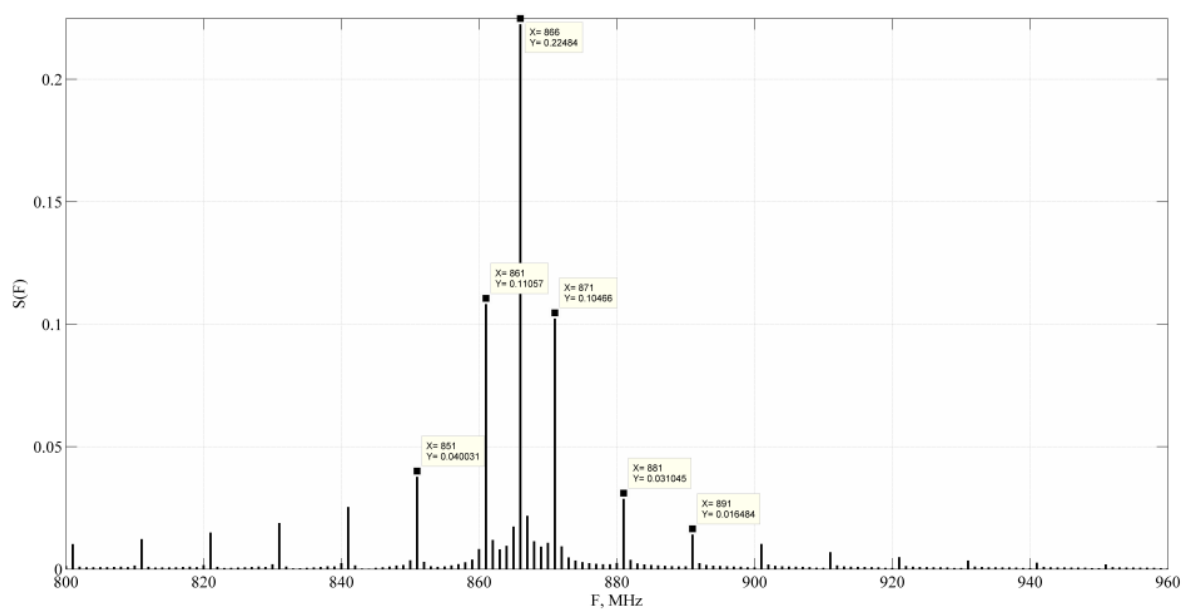

Fig. 4. Spectrum of the test sequence with ASK

Similarly, one can obtain time realizations and signal spectrum for other kinds of digitally modulated signals. But first, we have to determine the available values of the conjugate reflection coefficient. To expand the range of feasible values of the conjugate reflection coefficient we have to increase the input resistance $R_{I N}$ in 3.5 times and decrease the input capacitance $C_{I N}$ to $70 \%$ from the initial value of Monza 4QT chip for $866 \mathrm{MHz}$.

For these conditions, we present the comparison of modulation states for PSK-2 (Fig. 5) and power spectrum of the test sequence with PSK-2 (Fig. 6), QAM-4 (Fig. 7) and QAM-8 (Fig. 8). We have not shown the realization for the backscattering signal with FSK because this kind of modulation usually presented for DTS as kind of ASK or PSK with the varying pulse length $[12,13]$. Therefore, our method is applicable for FSK too. 


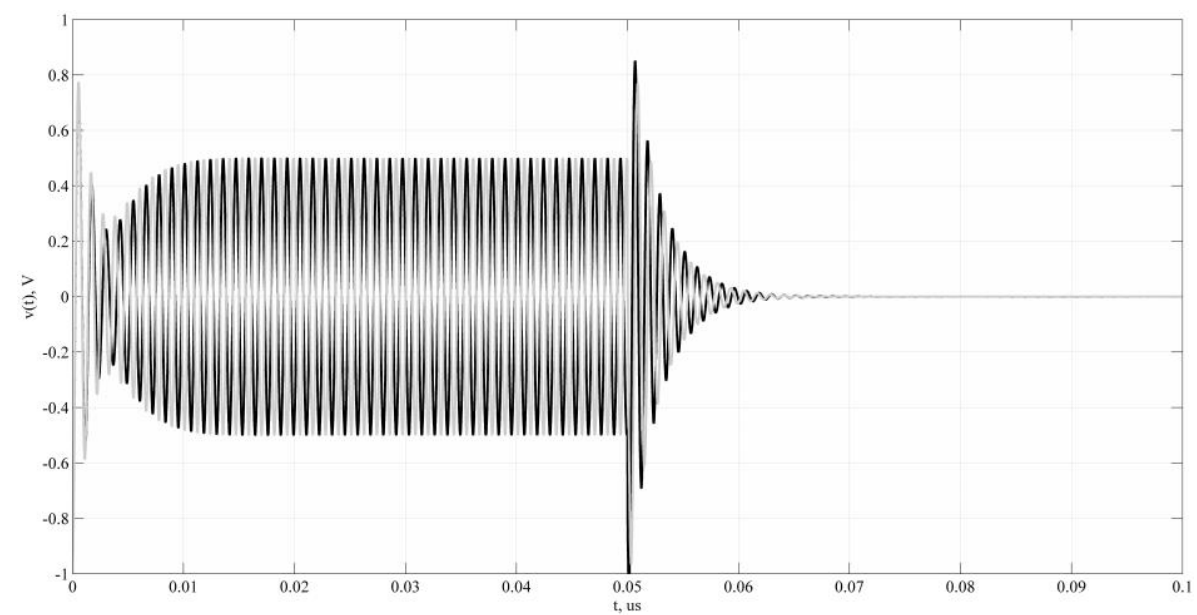

Fig. 5. Comparison of modulation states for PSK-2

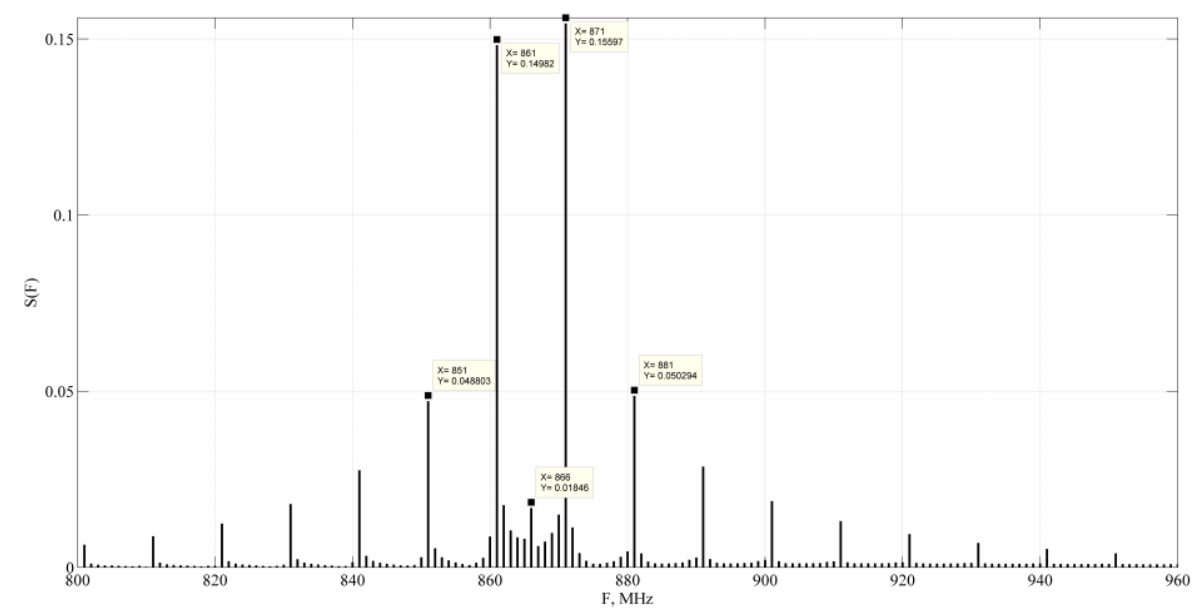

Fig. 6. Spectrum of the test sequence with PSK-2

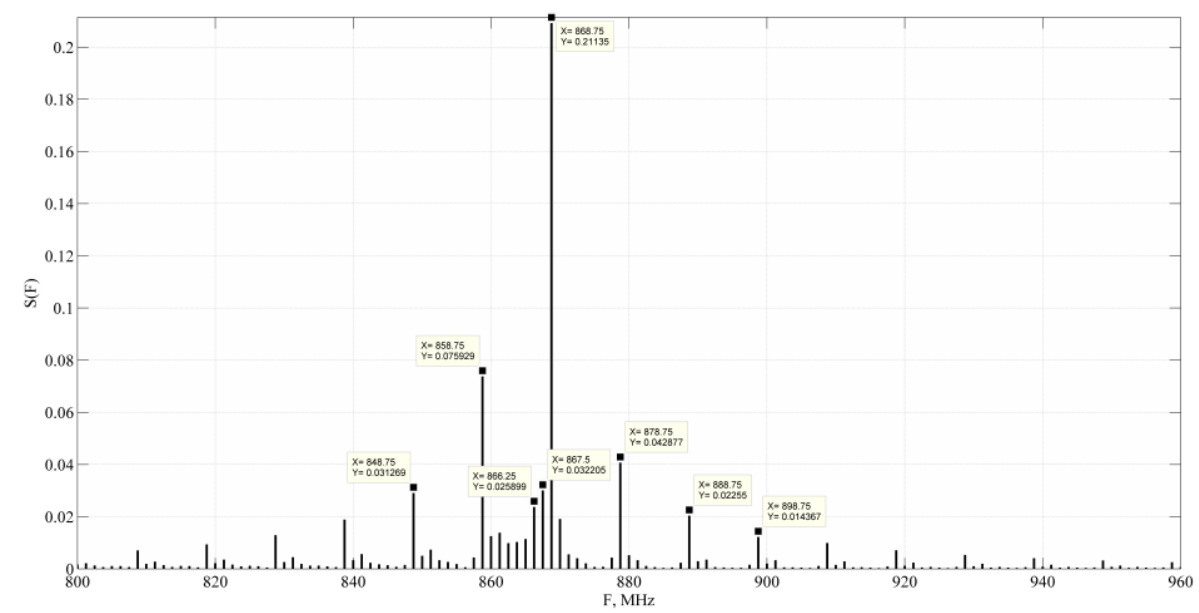

Fig. 6. Spectrum of the test sequence with QAM-4 


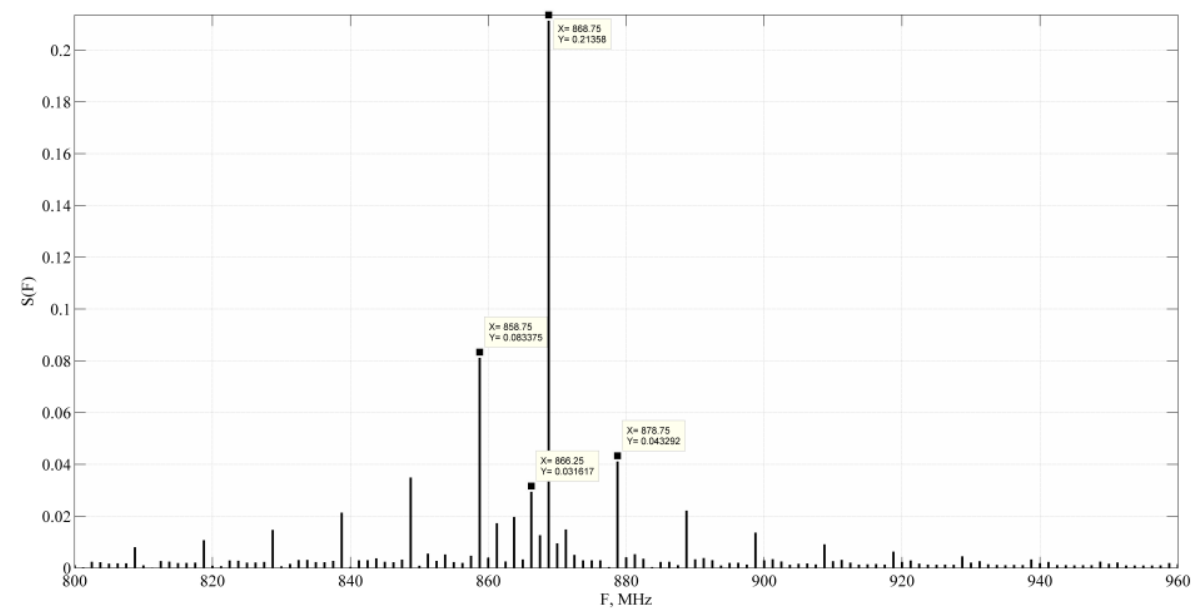

Fig. 6. Spectrum of the test sequence with QAM-8

\section{Conclusion}

Here we have presented the approach which is able to establish the connection between the law of the conjugate reflection coefficient of a tag and the necessary modulation type for the signal backscattered from a tag to a reader. We also showed the physical limitations on the choice of the conjugate reflection coefficient of a tag. The apparatus also allows us to model the influence of inertial properties of the tag's chain at the signal form.

As a confirmation of the possibility of using this approach for the purposes that have been identified above, we have modeled the signal conditioning for backscattered signals with ASK-2, PSK-2, QAM-4, QAM-8 modulation types.

\section{References}

1. F. Ensworth, S. Reynolds, IEEE RFID 2015, 78-85 (2015)

2. D. Bharadia, E. McMilin, S. Katti, SIGCOMM'15, 283-296 (2015)

3. V. Talla, S. Gollakota, J.R. Smith, NSDI'16, 151-164 (2016)

4. L. Shangguan, Y. Hu, Y. Qian, X. Lin, X. Chen, D. Fang, K. Jamieson, SIGCOMM'18, 147-160 (2018)

5. Y. Ma, N. Selby, F. Adib, SIGCOMM'17, 335-347 (2017)

6. V. Iyer, V. Talla, J.R. Smith, S. Gollakota, NSDI'17, 243-258 (2017)

7. S. Naderiparizi, M. Hessar, V. Talla, J.R. Smith, S. Gollakota, NSDI'18, 233-247 (2018)

8. O.V. Smirnov, E.A. Bogatyrev, REDS-2019, 128-133 (2019)

9. U. Karthaus, M. Fischer, IEEE JSSC, 38, 1602-1608 (2003)

10. S.J. Thomas, M.S. Reynolds, IEEE RFID 2012, 185-190 (2012)

11. O.V. Smirnov, E.A. Bogatyrev, S.M. Smolskiy, CriMiCo'2017, 300-306 (2017)

12. G. Vougioukas, A. Bletsas, WPTC, 1-3 (2016)

13. P. N. Alevizos, K. Tountas, A. Bletsas, TWC, $4522-4535$ (2018) 